\title{
New anti-inflammatory metabolites produced by Streptomyces violaceoruber isolated from Equus burchelli feces
}

\author{
Jian Ma ${ }^{1}$, Hui Lei ${ }^{1}$, Xiu Chen ${ }^{2}$, Xiaoxu Bi ${ }^{1}$, Yi Jiang ${ }^{2}$, Li Han ${ }^{1}$ and Xueshi Huang ${ }^{1}$ \\ Three new metabolites (2-4), together with one known compound, GTRI-02, (1) were isolated from a fermentation broth of \\ Streptomyces violaceoruber derived from Equus burchelli feces. The structures of the new compounds 2-4 were established using \\ comprehensive NMR spectroscopic data analysis as well as UV, IR and MS data. The anti-inflammatory activity of compounds 1-4 \\ was tested by examining their ability to inhibit nitric oxide (NO) production in lipopolysaccharide (LPS)-stimulated RAW 264.7 \\ macrophage cells. Compound 2 showed a moderate inhibition of NO production with $\mathrm{IC}_{50}$ value of $\mathbf{5 1 . 2} \boldsymbol{\mu \mathrm { M }}$.
}

The Journal of Antibiotics (2017) 70, 991-994; doi:10.1038/ja.2017.75; published online 12 July 2017

\section{INTRODUCTION}

Animal intestinal microorganisms have been studied for decades, and the investigation mainly focused on the food digestion and absorption, immunity, pathogens resistance and health maintaining. ${ }^{1}$ Over the past few years, the authors have dedicated to research on the diversity, bioactivities and the secondary metabolites of cultivable actinobacteria from animal feces and several new microbial species and new bioactive substances have been found. ${ }^{2-7}$ These findings showed animal intestinal and fecal microorganisms could be considered as a rich and important microbial resource for discovering new bioactive natural products.

In the course of our continuous search for potential structurally novel and bioactive natural products from fecal actinobacteria associated with animal feces, we investigated the secondary metabolites of Streptomyces violaceoruber (YIM 101131), which was isolated from Equus burchelli (zebra) feces. Separation of the fermentation broth of $S$. violaceoruber led to the isolation of three new compounds, a tetralone derivative, (R)-7-acetyl-3,6-dihydroxy-8-propyl-3,4dihydronaphthalen-1(2H)-one (2), a tryptophan derivative, $(S)-2-(5-$ (2,3-dihydroxy-3-methylbutyl)- $1 H$-indol-3-yl) acetonitrile (3), a fatty acid amide $\left(5 Z, 8 Z, 11 R^{*}, 12 R^{*}\right)$-11,12-dihydroxytetradeca-5,8-dienamide (4), as well as a known compound GTRI-02 $(\mathbf{1})^{8-10}$ (Figure 1). The structures of these metabolites were determined on the basis of comprehensive spectroscopic analysis and $\mathrm{Mo}_{2}(\mathrm{OAc})_{4}$ induced $\mathrm{CD}$ effects. The anti-inflammatory activity of isolated compounds 1-4 were evaluated by examining their ability to inhibit NO production in LPS-stimulated RAW 264.7 macrophage cells. Details of the isolation, structure elucidation and anti-inflammatory activity of the new compounds are described in the present paper.

\section{RESULTS AND DISCUSSION}

Compound 2 was isolated as a white, amorphous solid. The molecular formula of 2 was determined to be $\mathrm{C}_{15} \mathrm{H}_{18} \mathrm{O}_{4}$ based on the HRESIMS at $m / z 261.1218[\mathrm{M}-\mathrm{H}]^{-}$. The IR spectrum showed absorptions for hydroxyl $\left(3270 \mathrm{~cm}^{-1}\right)$, unsaturated carbonyl $\left(1698 \mathrm{~cm}^{-1}\right)$ and benzene ring $\left(1582 \mathrm{~cm}^{-1}\right)$. The ${ }^{1} \mathrm{H}$ NMR data (Table 1) of 1 displayed signals for an aromatic proton $\left(\delta_{\mathrm{H}} 6.59, \mathrm{~s}\right)$, an oxygenated methine proton $\left(\delta_{\mathrm{H}} 4.09, \mathrm{~m}\right)$ and two methyl groups at $\delta_{\mathrm{H}} 2.39(3 \mathrm{H}, \mathrm{s})$ and $\delta_{\mathrm{H}}$ $0.88(3 \mathrm{H}, \mathrm{t}, J=7.2 \mathrm{~Hz})$. Additional signals showed six aliphatic methylene protons $\left(\delta_{\mathrm{H}} 3.03, \mathrm{dd}, J=15.8,2.6 \mathrm{~Hz} ; \delta_{\mathrm{H}} 2.78\right.$, dd, $J=15.8,7.2 \mathrm{~Hz} ; \delta_{\mathrm{H}} 2.71$, brd, $J=16.0 \mathrm{~Hz} ; \delta_{\mathrm{H}} 2.46$, dd, $J=16.0,7.9$; $\left.\delta_{\mathrm{H}} 1.39,2 \mathrm{H}, \mathrm{m}\right)$. The ${ }^{13} \mathrm{C}$ NMR spectrum of 2 showed 15 carbon signals (Table 1). Except for the ${ }^{13} \mathrm{C}$ NMR signals corresponding with the ${ }^{1} \mathrm{H}$ NMR data, seven quaternary carbons, including two ketone carbonyls at $\delta_{\mathrm{C}} 205.8,196.8$, five aromatic quaternary carbons at $\delta_{\mathrm{C}}$ 159.1, 146.1, 142.9, 131.4, 122.1 were observed. Its ${ }^{1} \mathrm{H}$ and ${ }^{13} \mathrm{C} \mathrm{NMR}$ spectroscopic data of 2 were very similar to those of the known compound GTRI-02 (1), ${ }^{8-10}$ apart from the presence of two additional methylenes. Moreover, the ${ }^{1} \mathrm{H}-{ }^{1} \mathrm{H}$ COSY experiment gave a propyl fragment in 2. These findings suggested a propyl instead of a methyl located at C-8 in 2 . HMBC correlations between $\mathrm{H}-11\left(\delta_{\mathrm{H}} 2.72,2.66\right)$ and C-7 $\left(\delta_{\mathrm{C}} 131.4\right), \mathrm{C}-8\left(\delta_{\mathrm{C}} 142.9\right)$ and $\mathrm{C}-8 \mathrm{a}\left(\delta_{\mathrm{C}} 122.1\right)$, between $\mathrm{H}-12\left(\delta_{\mathrm{H}} 1.39\right)$ and C-8 $\left(\delta_{\mathrm{C}} 142.9\right)$ confirmed the propyl was linked to $\mathrm{C}-8$. The configuration of the chiral carbon $\mathrm{C}-3$ was assigned as $R$ by comparing the optical rotation value $\left([\alpha]_{D}^{20}-10(c 0.4, \mathrm{MeOH})\right)$ with that of $(R)$-GTRI-02 $\left([\alpha]_{D}^{20}-16(c\right.$ 2.5, MeOH $\left.)\right)$ which had been obtained by biomimetic asymmetric synthesis and the absolute configuration was determined by CD spectroscopy. ${ }^{11}$ Consequently,

${ }^{1}$ Institute of Microbial Pharmaceuticals, College of Life and Health Sciences, Northeastern University, Shenyang, PR China and ${ }^{2}$ Yunnan Institute of Microbiology, School of Life Science, Yunnan University, Kunming, PR China

Correspondence: Dr. Y Jiang, Yunnan Institute of Microbiology, School of Life Science, Yunnan University, Kunming 650091, PR China.

E-mail: jiangyi@ynu.edu.cn

or Professor L Han, Institute of Microbial Pharmaceuticals, College of Life and Health Sciences, Northeastern University, Wenhua Road 3-11, Shenyang 110819, PR China.

E-mail: hanli@mail.neu.edu.cn

Received 28 March 2017; revised 23 May 2017; accepted 27 May 2017; published online 12 July 2017 
<smiles>CC(=O)c1c(O)cc2c(c1C)C(=O)C[C@@H](O)C2</smiles><smiles>CCCc1c(C(C)=O)c(O)cc2c1C(=O)C[C@H](O)C2</smiles><smiles>CC(C)(O)C(O)Cc1ccc2[nH]cc(CC#N)c2c1</smiles><smiles>CCC(O)C(O)CC=CC=CCCCC(N)=O</smiles>

Figure 1 Structures of compounds $1-4$

Table $1{ }^{1} \mathrm{H}\left(600 \mathrm{MHz}\right.$, DMSO- $\left.d_{6}\right)$ and ${ }^{13} \mathrm{C}\left(150 \mathrm{MHz}\right.$, DMSO- $\left.d_{6}\right)$ NMR data of compound 2

\begin{tabular}{|c|c|c|c|}
\hline No. & $\delta_{C}$ & $\delta_{H}$ & $H M B C$ \\
\hline 1 & $196.8 \mathrm{C}$ & & \\
\hline 2 & $50.0 \mathrm{CH}_{2}$ & $\begin{array}{c}2.71, \text { brd }(16.0) \\
2.46, \text { dd }(16.0,7.9)\end{array}$ & $1,3,4,8 a$ \\
\hline 3 & $65.1 \mathrm{CH}$ & $4.09, \mathrm{~m}$ & \\
\hline 4 & $40.5 \mathrm{CH}_{2}$ & $\begin{array}{l}3.03 \text {, dd }(15.8,2.6) \\
2.78, \text { dd }(15.8,7.2)\end{array}$ & $2,3,4 a, 5,8 a$ \\
\hline $4 a$ & $146.1 \mathrm{C}$ & & \\
\hline 5 & $114.7 \mathrm{CH}$ & $6.59, \mathrm{~s}$ & $4,6,7,9,8 a$ \\
\hline 6 & $159.1 \mathrm{C}$ & & \\
\hline 7 & $131.4 \mathrm{C}$ & & \\
\hline 8 & $142.9 \mathrm{C}$ & & \\
\hline $8 a$ & $122.1 \mathrm{C}$ & & \\
\hline 9 & 205.8 C & & \\
\hline 10 & $32.9 \mathrm{CH}_{3}$ & $2.39, \mathrm{~s}$ & 7,9 \\
\hline 11 & $33.5 \mathrm{CH}_{2}$ & $\begin{array}{l}2.72, \mathrm{~m} \\
2.66, \mathrm{~m}\end{array}$ & $7,8,8 a, 12,13$ \\
\hline 12 & $25.0 \mathrm{CH}_{2}$ & $1.39, \mathrm{~m}$ & $8,11,13$ \\
\hline 13 & $15.0 \mathrm{CH}_{3}$ & $0.88, \mathrm{t}(7.2)$ & 11,12 \\
\hline
\end{tabular}

2 was identified as $(R)$-7-acetyl-3,6-dihydroxy-8-propyl-3,4-dihydronaphthalen-1(2H)-one.

Compound 3 was obtained as a colorless, amorphous solid. The HRESIMS exhibited a prominent ion peak at $\mathrm{m} / \mathrm{z} 257.1345[\mathrm{M}-\mathrm{H}]^{-}$, which established the molecular formula of 3 as $\mathrm{C}_{15} \mathrm{H}_{18} \mathrm{~N}_{2} \mathrm{O}_{2} \cdot{ }^{1} \mathrm{H}$ NMR spectrum (Table 2) of 3 indicated signals of four aromatic protons $\left(\delta_{\mathrm{H}} 7.41\right.$, brs; $\delta_{\mathrm{H}} 7.28, \mathrm{~d}, J=8.3 \mathrm{~Hz} ; \delta_{\mathrm{H}} 7.28$, brs; $\delta_{\mathrm{H}} 7.05, \mathrm{~d}$, $J=8.3 \mathrm{~Hz})$, one oxygenated methine $\left(\delta_{\mathrm{H}} 3.37, \mathrm{~m}\right)$, two methylene groups $\left(\delta_{\mathrm{H}} 4.00,2 \mathrm{H}, \mathrm{s} ; \delta_{\mathrm{H}} 3.02\right.$, brd, $J=13.6 \mathrm{~Hz} ; \delta_{\mathrm{H}} 2.43$, dd, $J=13.6$, $10.3 \mathrm{~Hz})$, two singlet methyls $\left(\delta_{\mathrm{H}} 1.14 ; \delta_{\mathrm{H}} 1.11\right)$. Moreover, three active protons were observed $\left(\delta_{\mathrm{H}} 10.96\right.$, brs; $\delta_{\mathrm{H}} 4.30, \mathrm{~d}, J=6.0 \mathrm{~Hz} ; \delta_{\mathrm{H}}$ $4.24, \mathrm{~s}) .{ }^{13} \mathrm{C}$ NMR spectrum (Table 2 ) of 3 presented 15 carbon signals including six carbons without directly bonded protons, five methines, two methylenes, two methyls with the aid of HSQC spectrum. These data showed 3 was a tryptophan derivative and related to 5-dimethylallylindole-3-acetonitrile. ${ }^{12}$ The major difference between these two compounds was the side chain at C-5. Two more oxygenated carbons were present and a double bond was absent in 3, which indicated the double bond in 5-dimethylallylindole-3acetonitrile was oxygenated to vicinal diol in 3 . The planar structure of 3 was further determined by $\mathrm{HMBC}$ correlations (Figure 2). The absolute configuration of C-9 was elucidated by $\mathrm{Mo}_{2}(\mathrm{AcO})_{4^{-}}$
Table $2{ }^{1} \mathrm{H}\left(600 \mathrm{MHz}\right.$, DMSO- $\left.\mathrm{d}_{6}\right)$ and ${ }^{13} \mathrm{C}\left(150 \mathrm{MHz}\right.$, DMSO- $\left.\mathrm{d}_{6}\right)$ NMR data of compound 3

\begin{tabular}{lccc}
\hline No. & $\delta_{\mathrm{C}}$ & $\delta_{H}$ & $H M B C$ \\
\hline 1 & & $10.96, \mathrm{~s}$ & $3,3 \mathrm{a}, 7 \mathrm{a}$ \\
2 & $124.2 \mathrm{CH}$ & $7.28, \mathrm{brs}$ & $3,7 \mathrm{a}$ \\
3 & $103.6 \mathrm{C}$ & & \\
$3 \mathrm{a}$ & $126.5 \mathrm{C}$ & $7.41, \mathrm{brs}$ & $3,6,7 \mathrm{a}, 1^{\prime}$ \\
4 & $118.4 \mathrm{CH}$ & & \\
5 & $132.2 \mathrm{C}$ & $7.05, \mathrm{brd}(8.3)$ & $4,7 \mathrm{a}$ \\
6 & $124.1 \mathrm{CH}$ & $7.28, \mathrm{~d}(8.3)$ & $3 a, 5$ \\
7 & $111.6 \mathrm{CH}$ & & $2,3,3 \mathrm{a}, 9$ \\
$7 \mathrm{a}$ & $135.3 \mathrm{C}$ & $4.00, \mathrm{~s}$ & \\
8 & $13.7 \mathrm{CH}$ & & $4,5,6,2^{\prime}$ \\
9 & $120.0 \mathrm{C}$ & $3.37, \mathrm{~m}$ & $5,3^{\prime}$ \\
$1^{\prime}$ & $38.1 \mathrm{CH}$ & $3.02, \mathrm{brd}(13.6) 2.43, \mathrm{dd}(13.6,10.3)$ & \\
$2^{\prime}$ & $80.1 \mathrm{CH}$ & & $2^{\prime}, 3^{\prime}, 5^{\prime}$ \\
$3^{\prime}$ & $72.3 \mathrm{C}$ & $1.11, \mathrm{~s}$ & $2^{\prime}, 3^{\prime}, 4^{\prime}$ \\
$4^{\prime}$ & $24.8 \mathrm{CH}_{3}$ & $1.14, \mathrm{~s}$ & $1^{\prime}, 2^{\prime}$ \\
$5^{\prime}$ & $27.2 \mathrm{CH}_{3}$ & $4.30, \mathrm{~d}(6.0)$ & $2^{\prime}, 3^{\prime}, 4^{\prime}$ \\
$2^{\prime}-\mathrm{OH}$ & & $4.24, \mathrm{~s}$ & \\
$3^{\prime}-\mathrm{OH}$ & & & \\
\hline
\end{tabular}
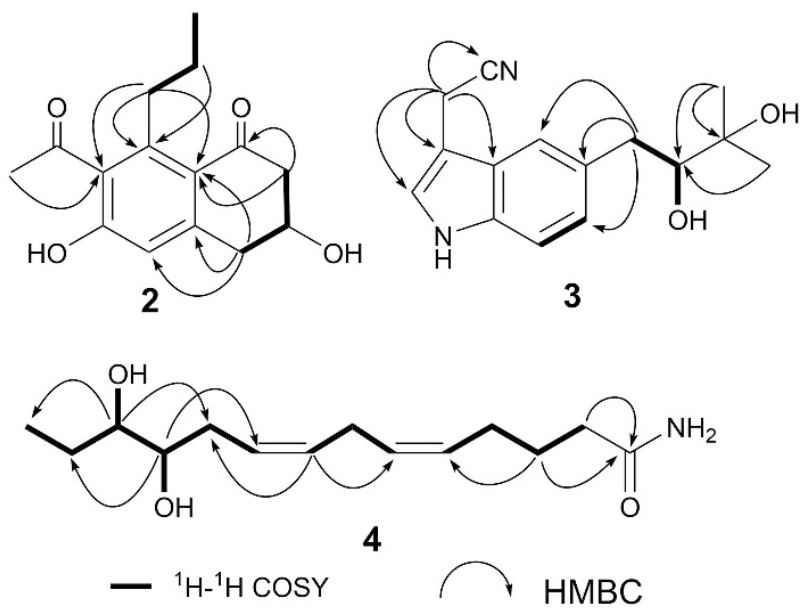

Figure $2{ }^{1} \mathrm{H}-{ }^{1} \mathrm{H}$ COSY and key HMBC correlations for compounds 2-4.

induced CD method. ${ }^{13}$ The CD spectrum showed a positive Cotton effect at $313 \mathrm{~nm}$ suggesting $\mathrm{S}$ configuration for C-2' (Figure 3). Thus, 3 was identified as (S)-2-(5-(2,3-dihydroxy-3methylbutyl)- $1 H$-indol-3-yl) acetonitrile.

Compound 4 was isolated as a white, amorphous solid. Its molecular formula was determined as $\mathrm{C}_{14} \mathrm{H}_{25} \mathrm{NO}_{3}$ by HRESIMS at $m / z 256.1992[\mathrm{M}+\mathrm{H}]^{+}$. The ${ }^{1} \mathrm{H}$ and ${ }^{13} \mathrm{C}$ NMR data indicated 4 was a fatty acid amide, consisting of 14 carbons including four olefinic carbons and two oxygenated carbons. HSQC experiment distinguished the protons linked to carbons. The uninterrupted ${ }^{1} \mathrm{H}-{ }^{1} \mathrm{H}$ COSY correlations from $\mathrm{H}-2$ to $\mathrm{H}_{3}-14$ determined the linkage of all the carbons. The planar structure of $\mathbf{4}$ was further confirmed by HMBC correlations from H-8 $\left(\delta_{\mathrm{H}} 5.49\right)$ to C-6 $\left(\delta_{\mathrm{C}} 128.9\right), \mathrm{C}-7\left(\delta_{\mathrm{C}} 25.9\right), \mathrm{C}-10$ $\left(\delta_{\mathrm{C}} 31.5\right)$, from H-11 $\left(\delta_{\mathrm{H}} 3.21\right)$ to $\mathrm{C}-9\left(\delta_{\mathrm{C}} 128.7\right), \mathrm{C}-12\left(\delta_{\mathrm{C}} 75.3\right)$, $\mathrm{C}-13\left(\delta_{\mathrm{C}} 25.9\right)$, from $\mathrm{H}-12\left(\delta_{\mathrm{H}} 3.12\right)$ to $\mathrm{C}-10\left(\delta_{\mathrm{C}} 31.5\right), \mathrm{C}-11$ $\left(\delta_{\mathrm{C}} 74.2\right), \mathrm{C}-14\left(\delta_{\mathrm{C}} 10.7\right)$, from $\mathrm{H}-3\left(\delta_{\mathrm{H}} 1.53\right)$ to $\mathrm{C}-1\left(\delta_{\mathrm{C}} 174.6\right)$, C-5 $\left(\delta_{\mathrm{C}} 129.7\right)$, from $\mathrm{H}-2\left(\delta_{\mathrm{H}} 2.04\right)$ to $\mathrm{C}-1\left(\delta_{\mathrm{C}} 174.6\right)$. Both the double 


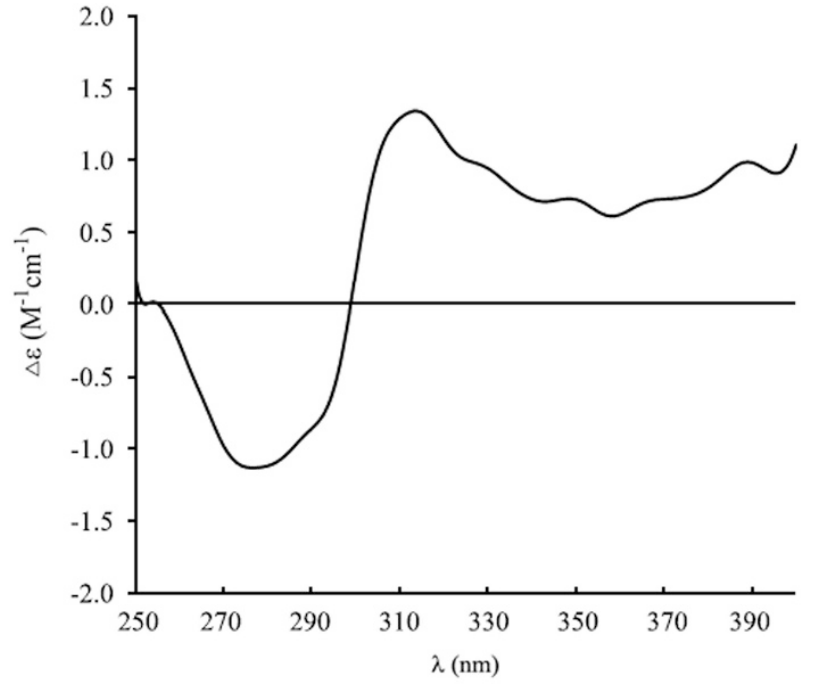

Figure $3 \mathrm{Mo}_{2}(\mathrm{AcO})_{4}$-induced $\mathrm{CD}$ spectrum of 3 .

bonds at C-5/C-6, C-8/C-9 elucidated as $Z$ geometry based on the coupling constant of $\mathrm{H}-8\left(\delta_{\mathrm{H}} 5.49, \mathrm{dt}, J=10.8,7.2 \mathrm{~Hz}\right)$ and the ${ }^{13} \mathrm{C}$ NMR data of C-7 $\left(\delta_{\mathrm{C}} 25.9\right) .{ }^{14,15}$ The relative configuration of C-11 and $\mathrm{C}-12$ was deduced as threo on the basis of the coupling constant ${ }^{3} J_{\mathrm{H}-11 / \mathrm{H}-12}=6.2 \mathrm{~Hz} .{ }^{16-18}$ Thus, the structure of 4 was established as (5Z, 8Z, $\left.11 R^{*}, 12 R^{*}\right)$-11,12-dihydroxytetradeca-5,8-dienamide.

Compounds 1-4 were tested for anti-inflammatory activity by examining their ability to inhibit NO production in LPS-stimulated RAW 264.7 macrophage cells. The effects of these compounds on cell viability were also determined by the MTT method, and none of these compounds exhibited cytotoxicities at $100 \mu \mathrm{M}$. Compared to the vehicle-treated group, compound 2 exhibited a moderate dose-dependent decrease in the NO production $(P<0.01)$ at the concentrations of 100, 33.3 and $11.1 \mu \mathrm{M}$ (Figure 4). While compounds 1, 3, 4 didn't show obvious inhibition at the tested concentrations. The $\mathrm{IC}_{50}$ value of compound 2 was further determined to be $51.2 \mu \mathrm{M}$. Minocycline was used as a positive control with $\mathrm{IC}_{50}$ value of $25.3 \mu \mathrm{M}$.

\section{METHODS}

\section{General experimental procedures}

Optical rotations were determined using an Anton Paar MCP200 automatic polarimeter (Graz, Austria). IR spectrum was recorded with a Bruker Tensor 27 FT-IR spectrometer (film). UV detection was carried out with a Beckman Coulter DU 730 nucleic acid/protein analyzer (Brea, CA, USA). CD spectra were acquired on a Biologic MOS-450 spectra polarimeter (Biologic Science, Claix, France). 1D and 2D NMR spectra were recorded on a Bruker AV-600 spectrometer (Faellanden, Switzerland). ESI-MS were recorded on an Agilent 1290-6420 Triple Quadrupole LC-MS spectrometer (Santa Clara, CA, USA). HRESI-MS was measured with an Agilent G6230 TOF mass spectrometer. Biological assays were analyzed using a microplate reader (BioTek Synergy H1, BioTek Instruments, Winooski, VT, USA).

\section{Microbial material}

The producing organism was isolated from fecal samples excreted by healthy adult E. burchelli living in Yunnan Wild Animal Park, Kunming, Yunnan province, China. The strain was identified as S. violaceoruber on the basis of morphological characteristics and $16 \mathrm{~S}$ ribosomal RNA gene sequences analysis. Phylogenetic analysis suggested that the strain shared a higher $16 \mathrm{~S}$ ribosomal RNA gene sequences similarity $(100 \%)$ with the closely related strain S. violaceoruber (accession number AB184174). The strain (No. YIM 101131)

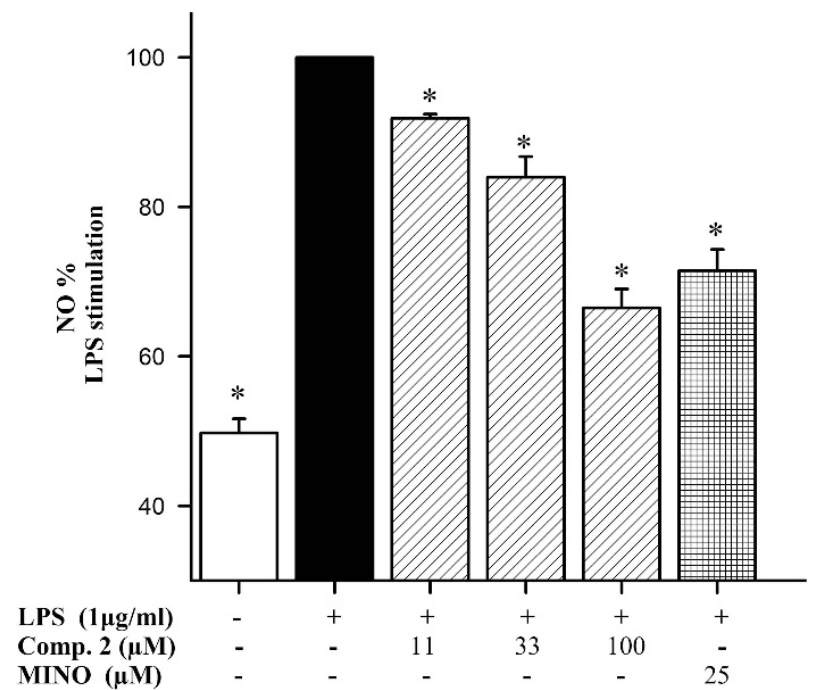

Figure 4 Effect of compound 2 on the inhibition of LPS-induced NO in Raw 264.7 cells.

was deposited at the Yunnan Institute of Microbiology, Yunnan University, China.

\section{Fermentation, extraction and isolation}

The strain, grown on agar plate, was prepared to inoculate $500 \mathrm{ml}$ Erlenmeyer flasks each containing $100 \mathrm{ml}$ of sterile seed medium composed of glucose $4.0 \%$, malt extract $5.0 \%$, multiple vitamin solution $1.0 \mathrm{ml}$ per liter and trace element solution $1.0 \mathrm{ml}$ per liter at a $\mathrm{pH}$ of 7.2 with no adjustment. These flasks cultures were incubated at $28^{\circ} \mathrm{C}$ for 2 days on a rotary shaker set at 180 r.p.m. For large-scale fermentation, $20 \mathrm{ml}$ of seed medium was used to transferred into $1000 \mathrm{ml}$ Erlenmeyer flasks containing $200 \mathrm{ml}$ of sterile fermentation medium composed of soybean meal $10 \mathrm{~g}$, peptone $2.0 \mathrm{~g}$, glucose $20 \mathrm{~g}$, soluble starch 5.0 g, yeast extract $2.0 \mathrm{~g}, \mathrm{NaCl} 4 \mathrm{~g}, \mathrm{~K}_{2} \mathrm{HPO}_{4} 0.5 \mathrm{~g}, \mathrm{MgSO}_{4} \cdot 7 \mathrm{H}_{2} \mathrm{O} 0.5 \mathrm{~g}$ and $\mathrm{CaCO}_{3} 2.0 \mathrm{~g}$ in 11 tap water at a $\mathrm{pH}$ of 7.8 with no adjustment. The fermentation batches were cultured at $28^{\circ} \mathrm{C}$ for 7 days on a rotary shaker set at 180 r.p.m.

The mycelium and broth filtrate $(70 \mathrm{l})$ were separated by centrifugation (4000 r.p.m, $5 \mathrm{~min}$ ). The resultant aqueous phase filtrate was extracted with EtOAc for three times. The organic partition layer was collected and evaporated in vacuo to yield $13 \mathrm{~g}$ of residue. The dried crude extract was further separated by chromatography on a silica gel column $\left(\mathrm{CH}_{2} \mathrm{Cl}_{2}-\mathrm{MeOH}\right.$, gradient $\left.50: 1-1: 1\right)$ to obtain 10 fractions (Fr. 1-10). Fraction 4 was subjected to Sephadex LH-20 chromatography $(\mathrm{MeOH})$ to afford six subfractions (Fr. 4.1-4.6). Fraction 4.3 was purified using semi-preparative reversed-phase HPLC $\left(\mathrm{MeOH}-\mathrm{H}_{2} \mathrm{O} 55: 45\right.$, $\left.10 \mathrm{ml} \mathrm{min}^{-1}\right)$ to yield compound $3(5.5 \mathrm{mg})$. Fraction 8 was subjected to Sephadex LH-20 chromatography $(\mathrm{MeOH})$ to produce six subfractions (Fr. 8.1-8.6). Subfraction 8.5 was applied to semi-preparative reversed-phase

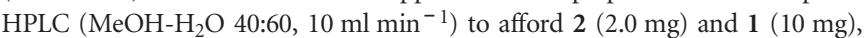
respectively. Fraction 10 was separated by silica gel column chromatography (petroleum ether-EtOAc 1:5) to afford six subfractions (Fr. 10.1-10.6). Subfraction 10.2 was purified by semi-preparative reversed-phase HPLC $\left(\mathrm{MeOH}-\mathrm{H}_{2} \mathrm{O} 57: 43,10 \mathrm{ml} \mathrm{min}^{-1}\right)$ to yield $4(4.2 \mathrm{mg})$.

(R)-7-acetyl-3,6-dihydroxy-8-propyl-3,4-dihydronaphthalen-1(2H)-one (2): white, amorphous solid; $[\alpha]_{D}^{20}-10(c 0.4, \mathrm{MeOH}) ; \mathrm{UV}(\mathrm{MeOH}) \lambda_{\max }(\log \varepsilon)$ 205 (4.40), 275 (4.22) nm; IR (film) $\nu_{\max } 3270,2959,2927,1698,1667,1582$, 1276, 1236, 1211, $1026 \mathrm{~cm}^{-1}$; ESI-MS $\mathrm{m} / \mathrm{z} 263[\mathrm{M}+\mathrm{H}]^{+}, 285[\mathrm{M}+\mathrm{Na}]^{+}$; HRESI-MS $m / z 263.1286[\mathrm{M}+\mathrm{H}]^{+}\left(\right.$calcd for $\left.\mathrm{C}_{15} \mathrm{H}_{19} \mathrm{O}_{4}, 263.1283\right)$. For ${ }^{1} \mathrm{H}$ and ${ }^{13} \mathrm{C}$ NMR data see Table 1.

(S)-2-(5-(2,3-dihydroxy-3-methylbutyl)-1H-indol-3-yl) acetonitrile (3): colorless, amorphous solid; $[\alpha]_{D}^{20}-51.8($ c $0.54, \mathrm{MeOH})$; UV $(\mathrm{MeOH}) \lambda_{\max }$ (log $\varepsilon) 219$ (4.48), 274 (3.72) nm; IR (film) $\nu_{\max }$ 3351, 2971, 2923, 2251, 1660, $1604,1429,1381,1348,1069 \mathrm{~cm}^{-1} ; \mathrm{Mo}_{2}(\mathrm{OAc})_{4}$ induced $\mathrm{CD} \lambda_{\max }(\Delta \varepsilon) 313$ 
(1.34); ESI-MS $m / z 281[\mathrm{M}+\mathrm{Na}]^{+}$; HRESI-MS $m / z 281.1265[\mathrm{M}+\mathrm{Na}]^{+}$(calcd for $\mathrm{C}_{15} \mathrm{H}_{18} \mathrm{~N}_{2} \mathrm{O}_{2} \mathrm{Na}$, 281.1266); For ${ }^{1} \mathrm{H}$ and ${ }^{13} \mathrm{C}$ NMR data see Table 2.

(5Z, 8Z, $\left.11 R^{*}, 12 R^{*}\right)$-11,12-dihydroxytetradeca-5,8-dienamide (4): white, amorphous solid; $[\alpha]_{D}^{20}-25$ (c 0.36, MeOH); UV (MeOH) $\lambda_{\max }(\log \varepsilon) 219$ (3.90) nm; IR (film) $\nu_{\max } 3331,2927,1662,1612,1405 \mathrm{~cm}^{-1}$; ESI-MS m/z 256 $[\mathrm{M}+\mathrm{H}]^{+}, 278[\mathrm{M}+\mathrm{Na}]^{+}$; HRESI-MS $m / z 278.1719[\mathrm{M}+\mathrm{Na}]^{+}$(calcd for $\left.\mathrm{C}_{14} \mathrm{H}_{25} \mathrm{NO}_{3} \mathrm{Na}, 278.1732\right) ;{ }^{1} \mathrm{H}$ NMR $\left(600 \mathrm{MHz}\right.$, DMSO- $\left.d_{6}\right) \delta: 7.23(1 \mathrm{H}$, brs, $\mathrm{NH}), 6.69(1 \mathrm{H}$, brs, NH), $5.49(1 \mathrm{H}, \mathrm{dt}, J=10.8,7.2 \mathrm{~Hz}, \mathrm{H}-8), 5.34(3 \mathrm{H}, \mathrm{m}$, H-5, H-6, H-9), $4.40(1 \mathrm{H}, \mathrm{d}, J=5.9 \mathrm{~Hz}, 11-\mathrm{OH}), 4.33(1 \mathrm{H}, \mathrm{d}, J=5.9 \mathrm{~Hz}$, 12- $\mathrm{OH}), 3.21$ (1H, m, H-11), 3.12 (1H, m, H-12), 2.75 (2H, m, H-7), 2.35 (1H, m, H-10), $2.04(2 \mathrm{H}, \mathrm{t}, J=7.5 \mathrm{~Hz}, \mathrm{H}-2), 2.01$ (3H, m, H-10, H-4), 1.58 $(1 \mathrm{H}, \mathrm{m}, \mathrm{H}-13), 1.53(2 \mathrm{H}, \mathrm{m}, \mathrm{H}-3), 1.25(1 \mathrm{H}, \mathrm{m}, \mathrm{H}-13), 0.87(3 \mathrm{H}, \mathrm{t}, J=7.5 \mathrm{~Hz}$, H-14); ${ }^{13} \mathrm{C}$ NMR (150 MHz, DMSO- $\left.d_{6}\right) \delta: 174.6(\mathrm{C}-2, s), 35.1(\mathrm{C}-3, \mathrm{t}), 25.6$ (C-4, t), 26.8 (C-5, t), 129.7 (C-5, d), 128.9 (C-6, d), 25.9 (C-7, t), 128.3 (C-8, d), 128.7 (C-9, d), 31.5 (C-10, t), 74.2 (C-11, d), 75.3 (C-12, d), 25.9 (C-13, t), 10.7 (C-14, q). ${ }^{1} \mathrm{H}$ NMR $\left(600 \mathrm{MHz}, \mathrm{CD}_{3} \mathrm{OD}\right) \delta: 5.42(1 \mathrm{H}$, brdt, $J=10.8$, $7.3 \mathrm{~Hz}, \mathrm{H}-9), 5.34$ (1H, brdt, $J=10.8,7.3 \mathrm{~Hz}, \mathrm{H}-8), 5.32-5.26(2 \mathrm{H}, \mathrm{m}, \mathrm{H}-5$, $\mathrm{H}-6), 3.31(1 \mathrm{H}, \mathrm{ddd}, J=8.6,6.2,3.7 \mathrm{~Hz}, \mathrm{H}-11), 3.23$ (1H, m (overlapped), H-12), 2.74 (2H, m, H-7), 2.35 (1H, brddd, $J=14.9,7.3,3.7 \mathrm{~Hz}, \mathrm{H}-10), 2.12$ $(2 \mathrm{H}, \mathrm{t}, J=7.3 \mathrm{~Hz}, \mathrm{H}-2), 2.07$ (1H, ddd (quint-like), $J=14.9,8.6,7.3 \mathrm{~Hz}, \mathrm{H}-10$ ), $2.02(2 \mathrm{H}, \mathrm{q}, J=7.3 \mathrm{~Hz}, \mathrm{H}-4), 1.60(1 \mathrm{H}, \mathrm{m}, \mathrm{H}-13), 1.57(2 \mathrm{H}$, quint, $J=7.3 \mathrm{~Hz}$, H-3), 1.29 (1H, m, H-13), 0.89 (3H, t, J=7.3 Hz, H-14).

\section{Inhibition of NO production assay}

The nitrite concentration in the medium was measured using the Griess reaction as previously described. ${ }^{19}$ Compounds 1-4 were tested for their inhibitory activity of NO production at various concentrations (100, 33.3 and $11.1 \mu \mathrm{M}$ for dose-dependent assay; 10, 20, 40, 60, 80 and $100 \mu \mathrm{M}$ for $\mathrm{IC}_{50}$ analysis). Minocycline was used as the positive control. Statistical analysis was performed by SPSS (SPSS Inc., Chicago, IL, USA).

\section{CONFLICT OF INTEREST}

The authors declare no conflict of interest.

\section{ACKNOWLEDGEMENTS}

This work was funded by National Natural Science Foundation of China (Nos 81573327 and 31460005), Basic Scientific Research Fund of Northeastern University, China (No N142002001).
1 Savage, D. C. Microbial ecology of the gastrointestinal tract. Annu. Rev. Microbiol. 31, 107-133 (1977).

2 Jiang, Y. et al. Diversity and bioactivity of cultivable animal fecal actinobacteria. Adv. Microbiol. 3, 1-13 (2013).

3 Jiang, Y. et al. Diversity of cultivable actinomycetes in 6 species of herbivore feces. Int. J. Microbiol. Res. 2, 110-117 (2013).

4 Chen, X. et al. Enteractinococcus lamae sp. nov. and Enteractinococcus viverrae sp. nov., isolated from animal faeces. Antonie Van Leeuwenhoek 108, 1477-1483 (2015).

5 Chen, X. et al. Corynebacterium faecale sp. nov. isolated from the faeces of Assamese macaque. Int. J. Syst. Evol. Microbiol. 66, 2478-2483 (2016).

6 Zhang, J. et al. Violapyrones A-G, $\alpha$-pyrone derivatives from Streptomyces violascens isolated from Hylobates hoolock feces. J. Nat. Prod. 76, 2126-2130 (2013).

7 Ding, N. et al. Bafilomycins and odoriferous sesquiterpenoids from Streptomyces albolongus isolated from Elephas maximus feces. J. Nat. Prod. 79, 799-805 (2016).

8 Yeo, W. H. et al. GTRI-02, a new lipid peroxidation inhibitor from Micromonospora sp. SA246. J. Antibiot. 51, 952-953 (1998).

9 Abdalla, M. A. et al. Khatmiamycin, a motility inhibitor and zoosporicide against the grapevine downy mildew pathogen Plasmopara viticola from Streptomyces sp. ANK313. J. Antibiot. 64, 655-659 (2011).

10 Maskey, R. P. et al. Juglomycins G-J: isolation from streptomycetes and structure elucidation. Z. Naturforsch. B 60, 183-188 (2005).

11 Husain, S. M., Schätzle, M. A., Röhr, C., Lüdeke, S. \& Müller, M. Biomimetic asymmetric synthesis of (R)-GTRI-02 and (3 $S, 4 R$ )-3,4-dihydroxy-3,4-dihydronaphthalen-1(2H)-ones. Org. Lett. 14, 3600-3603 (2012).

12 Ozaki, T., Nishiyama, M. \& Kuzuyama, T. Novel tryptophan metabolism by a potential gene cluster that is widely distributed among actinomycetes. J. Biol. Chem. 288, 9946-9956 (2013).

13 Bari, L. D., Pescitelli, G., Pratelli, C., Pini, D. \& Salvadori, P. Determination of absolute configuration of acyclic 1,2-diols with $\mathrm{Mo}_{2}(\mathrm{OAc})_{4}$. 1. Snatzke's method revisited. J. Org. Chem. 66, 4819-4825 (2001).

14 Zhang, H., Xiao, X., Conte, M. M., Khalil, Z. \& Capon, R. J. Spiralisones A-D: acylphloroglucinol hemiketals from an Australian marine brown alga Zonaria spiralis. Org. Biomol. Chem. 10, 9671-9676 (2012).

$15 \mathrm{Liu}, \mathrm{F}$. et al. Phloroglucinol derivatives with protein tyrosine phosphatase 1B inhibitory activities from Eugenia jambolana seeds. J. Nat. Prod. 80, 544-550 (2017).

$16 \mathrm{Wu}, \mathrm{Q}$. et al. Varioxiranols A-G and 19-0-methyl-22-methoxypre-shamixanthone, PKS and hybrid PKS-derived metabolites from a sponge-associated Emericella variecolor fungus. J. Nat. Prod. 78, 2461-2470 (2015).

17 Jarvis, B. B., Wang, S. \& Ammon, H. L. Trichoverroid stereoisomers. J. Nat. Prod. 59, 254-261 (1996).

18 Du, L., Zhu, T., Fang, Y., Gu, Q. \& Zhu, W. Unusual C25 steroid isomers with bicyclo [4.4.1] A/B rings from a volcano ash-derived fungus Penicillium citrinum. J. Nat. Prod. 71, 1343-1351 (2008)

$19 \mathrm{Ma}, \mathrm{Q}$. et al. Structures and biological activities of the triterpenoids and sesquiterpenoids from Alisma orientale. Phytochemistry 131, 150-157 (2016).

Supplementary Information accompanies the paper on The Journal of Antibiotics website (http://www.nature.com/ja) 